\title{
Crescimento e produtividade de juvenis de robalo-peva a diferentes temperaturas e taxas de alimentação
}

\author{
Luiz Augusto Altenburg Gomes Oliveira(1), André Marafon Almeida( ${ }^{(2)}$, Pablo Seagan Vaz Pandolfo(2), \\ Rodrigo Matos de Souza(2), Luiz Fernando Loureiro Fernandes ${ }^{(2)}$ e Levy Carvalho Gomes ${ }^{(1)}$
}

\begin{abstract}
(1)Universidade Vila Velha, Rua Comissário José Dantas de Melo, no 21, Boa Vista, CEP 22102-770 Vila Velha, ES E-mail: laago.ppee2010@gmail.com, levy.gomes@uvv.br (2)Universidade Federal do Espírito Santo, Base Oceanográfica, Rodovia ES-010, Km 16, no 565, Santa Cruz, CEP 29190-000 Aracruz, ES. E-mail: decoctba@hotmail.com, wavesiegan@gmail.com, rodrigoms_uca@ymail.com, luiz.ufes@gmail.com
\end{abstract}

Resumo - O objetivo deste trabalho foi avaliar o efeito da temperatura e da taxa de alimentação sobre o crescimento e a produtividade de juvenis de robalo-peva (Centropomus parallelus). Utilizou-se experimento fatorial com duas temperaturas $\left(25\right.$ e $\left.28^{\circ} \mathrm{C}\right)$ e duas taxas de alimentação ( 3 e $6 \%$ da biomassa ao dia), com três repetições para cada combinação de temperatura e taxa. Os peixes $(9,80 \pm 0,41 \mathrm{~g} ; 9,86 \pm 0,14 \mathrm{~cm})$ foram alimentados duas vezes ao dia durante 60 dias. A cada 15 dias, foram realizadas amostragens para corrigir a quantidade de ração oferecida. Não houve mortalidade durante o experimento. Maior crescimento em peso e comprimento foi obtido nos animais submetidos à temperatura de $28^{\circ} \mathrm{C}$. Ganho de peso, biomassa final, conversão alimentar aparente e taxa de crescimento específico foram influenciados pela taxa de alimentação e pela temperatura. A oferta de $3 \%$ da biomassa ao dia a $28^{\circ} \mathrm{C}$ proporciona melhores índices de crescimento e produtividade para juvenis de robalo-peva.

Termos para indexação: Centropomus parallelus, aquicultura, bem-estar animal, desempenho produtivo, qualidade da água.

\section{Growth and yield of juvenile fat snook at different temperatures and feeding rates}

\begin{abstract}
The objective of this work was to evaluate the effects of temperature and feeding rates on growth and productivity of juvenile fat snook (Centropomus parallelus). A factorial experiment with two temperatures $\left(25\right.$ and $28^{\circ} \mathrm{C}$ ) and two feeding rates $(3$ and $6 \%$ of biomass per day) was used, with three replicates for each combination of temperature and rate. Fish $(9.80 \pm 0.41 \mathrm{~g} ; 9.86 \pm 0.14 \mathrm{~cm})$ were fed twice a day during 60 days. Every 15 days, samples were taken to correct the amount of food offered. No mortality was observed during the experiment. A greater growth in weight and length was obtained in animals subjected to the temperature of $28^{\circ} \mathrm{C}$. Weight gain, final biomass, apparent food conversion, and specific growth rate were influenced by feeding rate and temperature. The offer of $3 \%$ of biomass per day at $28^{\circ} \mathrm{C}$ provides better growth and productivity indices for juvenile fat snook.
\end{abstract}

Index terms: Centropomus parallelus, aquaculture, animal welfare, productive performance, water quality.

\section{Introdução}

O robalo-peva [Centropomus parallelus (Poey, 1860)] é um dos principais candidatos para o desenvolvimento da piscicultura em água salgada e salobra no Brasil. Há grande conhecimento sobre sua reprodução em cativeiro (Cerqueira \& Tsuzuki, 2009), e esta é uma das únicas espécies nativas com a qual é possível realizar a produção de juvenis em massa (Alvarez-Lajonchère et al., 2002), fator primordial para o desenvolvimento de uma espécie. Características como hábito gregário, robustez, resistência a doenças, tolerância a altas densidades de estocagem e a amplas variações de salinidade, bem como alto valor de mercado (Tsuzuki et al., 2007; Alvarez-Lajonchère \& Tsuzuki, 2008; Ribeiro \& Tsuzuki, 2010), têm estimulado o interesse na criação da espécie.

$\mathrm{O}$ robalo-peva ocorre em áreas tropicais e subtropicais, na costa atlântica das Américas, desde o litoral da Carolina do Norte, nos Estados Unidos da América, até o Rio Grande do Sul, no Brasil (Rivas, 1986), em faixa de temperatura bastante variável. Cerqueira (2010) registrou baixo crescimento de juvenis de robalo-peva a $22^{\circ} \mathrm{C}$. Ferraz et al. (2011) 
relataram que a melhor temperatura para o crescimento de larvas de robalo-peva é $30^{\circ} \mathrm{C}$. De acordo com Xie et al. (2011), a temperatura da água é um dos fatores ambientais que mais afetam as respostas fisiológicas dos peixes quanto ao crescimento e à alimentação.

Para diversas espécies de peixes tropicais, como o tambaqui [Colossoma macropomum (Cuvier, 1816)] (Silva et al., 2007) e o "catfish" chinês [Clarias fuscus (Lacepède, 1803)] (Anderson \& Fast, 1991), é comum a oferta de alimento a uma taxa de $5-10 \%$ do peso vivo ao dia durante a primeira fase do crescimento. O robalo-peva é um peixe voraz, que aumenta o consumo de alimento com o aumento da temperatura (Cerqueira, 2010). Este padrão está relacionado ao metabolismo dos peixes, cuja velocidade aumenta de forma proporcional ao aumento da temperatura (Baldisserotto, 2009). A oferta da ração em quantidade adequada é importante, pois o custo com alimentação artificial pode representar mais de $60 \%$ do custo variável em cultivos intensivos ou superintensivos (Silva et al., 2007).

O objetivo deste trabalho foi avaliar o efeito da temperatura e da taxa de alimentação no crescimento e na produtividade de juvenis de robalo-peva.

\section{Material e Métodos}

Juvenis de robalo-peva $(9,80 \pm 0,41 \mathrm{~g} ; 9,86 \pm 0,14 \mathrm{~cm})$ foram obtidos do laboratório de larvicultura marinha, Maricultura Pandini, São Mateus, ES, e alocados em 12 caixas de plástico de $400 \mathrm{~L}$, em densidades de 60 peixes por caixa (150 peixes por metro cúbico). As caixas eram abastecidas individualmente e continham sistema de aeração constante por pedra porosa e termostato de $400 \mathrm{~W}$, para manutenção da temperatura. A salinidade durante todo o experimento foi mantida em 5 ppt por meio da mistura de água marinha (32 ppt) e água doce sem cloro (Rocha et al., 2005). O sistema foi mantido estático, com troca de $80 \%$ da água das caixas duas vezes por semana.

Foi realizado experimento fatorial com duas taxas de alimentação diária ( 3 e $6 \%$ da biomassa ao dia) e duas temperaturas da água $\left(25\right.$ e $\left.28^{\circ} \mathrm{C}\right)$, com três repetições para cada combinação. A ração (NRD INVE, $1,2 \mathrm{~mm}$, com $59 \%$ de proteína bruta e $82 \%$ de energia metabolizável) foi ofertada em duas refeições por dia (às 9 e às 16h). O experimento teve duração de 60 dias.
A cada 15 dias, $50 \%$ dos animais de cada caixa eram capturados, anestesiados com $25 \mathrm{mg} \mathrm{L}^{-1}$ de eugenol e pesados. Após cada etapa de amostragem, o total de alimento ofertado aos animais era corrigido de acordo com a biomassa em cada caixa experimental. Com os resultados da última amostragem, foram calculados o crescimento em peso e comprimento total, e a taxa de crescimento específico: $\mathrm{TCE}=100[$ ( $\ln$ peso final médio - ln peso inicial médio)/tempo]. Ao final do experimento, os seguintes parâmetros de produção foram avaliados: sobrevivência (\%), ganho de peso $(\mathrm{GP}=$ peso final - peso inicial $)$, biomassa final $(\mathrm{BF}=$ biomassa final x sobrevivência) e conversão alimentar aparente (CAA = consumo de ração/ganho de peso).

Foram avaliados, três vezes por semana, o oxigênio dissolvido, a temperatura e a salinidade com oxímetro digital (YSI 85, YSI Incorporated, Yellow Springs, OH, EUA); e a cada sete dias, a amônia total foi determinada pelo método do azul de endofenol, conforme American Public Health Association (1992).

Os resultados foram analisados por análise de variância de dois fatores (taxa de alimentação e temperatura) e pelo teste de Tukey, a 5\% de probabilidade (Zar, 1999).

\section{Resultados e Discussão}

Não houve efeito da taxa de alimentação e da temperatura sobre a sobrevivência dos animais, que foi de $100 \%$ para todos os tratamentos. Esse resultado corrobora o obtido por Cerqueira \& Tsuzuki (2009), que observaram que esta espécie é resistente a condições de cultivo e a diferentes parâmetros ambientais.

Nos parâmetros ambientais analisados, o oxigênio dissolvido variou de 7,4 $\pm 0,1$ a $9,7 \pm 0,4 \mathrm{mg} \mathrm{L}^{-1}$ e diferiu significativamente entre as temperaturas, a uma mesma taxa de alimentação, e entre as diferentes taxas de alimentação, a uma mesma temperatura (Tabela 1). A salinidade manteve-se estável durante todo o experimento. A amônia total apresentou diferenças significativas entre as diferentes taxas de alimentação. O maior valor médio foi de $1,29 \pm 0,1 \mathrm{mg} \mathrm{L}^{-1}$, no tratamento $6 \%$ da biomassa ao dia a $25^{\circ} \mathrm{C}$, e o menor foi de $0,72 \pm 0,1 \mathrm{mg} \mathrm{L}^{-1}$, no tratamento $3 \%$ da biomassa ao dia a $28^{\circ} \mathrm{C}$. Com exceção da amônia, os demais parâmetros de qualidade da água mensurados estiveram dentro da faixa adequada para a criação de robalo-peva, de acordo com Cerqueira (2010). A concentração de 
amônia nos tanques dos animais alimentados com $6 \%$ da biomassa ao dia foi cerca de 50-60\% mais alta do que a dos tanques dos animais alimentados com 3\% da biomassa ao dia, independentemente da temperatura. Não se conhece o efeito da amônia no crescimento do robalo-peva, mas é possível que as maiores concentrações encontradas nos tanques dos animais alimentados com $6 \%$ da biomassa ao dia $\left(<1,5 \mathrm{mg} \mathrm{L}^{-1}\right.$ de amônia total) tenham sido uma das razões para o menor crescimento dos peixes, como sugerido por Lemarié et al. (2004) para juvenis de robalo europeu (Dicentrarchus labrax). Além disso, essa concentração é próxima à concentração tóxica aguda para peixes marinhos, que é de $1,86 \mathrm{mg} \mathrm{L}^{-1}$ (Randall \& Tissui, 2002).

Maiores peso e comprimento finais foram obtidos nos animais submetidos à temperatura de $28^{\circ} \mathrm{C}$ do que nos submetidos a $25^{\circ} \mathrm{C}$ (Tabela 2). Para os peixes criados na maior temperatura, não houve

Tabela 1. Parâmetros físico-químicos de qualidade de água (média \pm desvio-padrão) utilizados no experimento com robalo-peva (Centropomus parallelus) durante a primeira fase de crescimento, em diferentes taxas de alimentação e temperaturas.

\begin{tabular}{lcccc}
\hline $\begin{array}{l}\text { Temperatura } \\
\left({ }^{\circ} \mathrm{C}\right)\end{array}$ & $\begin{array}{c}\text { Oxigênio dissolvido } \\
\left(\mathrm{mg} \mathrm{L}^{-1}\right)\end{array}$ & $\begin{array}{c}\text { Temperatura } \\
\left({ }^{\circ} \mathrm{C}\right)\end{array}$ & $\begin{array}{c}\text { Salinidade } \\
(\mathrm{ppt})\end{array}$ & $\begin{array}{c}\text { Amônia } \\
\left(\mathrm{mg} \mathrm{L}^{-1}\right)\end{array}$ \\
\hline \multicolumn{5}{c}{$\begin{array}{c}3 \% \text { da biomassa ao dia } \\
25\end{array}$} \\
28 & $9,7 \pm 0,4^{*+}$ & $24,9 \pm 0,1^{*}$ & $5,1 \pm 0,1$ & $0,79 \pm 0,2^{+}$ \\
& $8,0 \pm 0,2^{+}$ & $27,9 \pm 0,1$ & $5,1 \pm 0,1$ & $0,72 \pm 0,1^{+}$ \\
25 & $9,0 \pm 0,2^{*}$ & $6 \%$ da biomassa ao dia \\
28 & $7,4 \pm 0,1$ & $24,8 \pm 0,1^{*}$ & $5,1 \pm 0,1$ & $1,29 \pm 0,1$ \\
\hline
\end{tabular}

*Diferença entre as diferentes temperaturas em uma mesma taxa de alimentação, a $5 \%$ de probabilidade. ${ }^{+}$Diferença significativa entre taxas de alimentação em uma mesma temperatura, a $5 \%$ de probabilidade. diferença significativa entre as taxas de alimentação, enquanto aqueles alimentados a $25^{\circ} \mathrm{C}$ com $3 \%$ da biomassa ao dia apresentaram peso e comprimento significativamente maiores do que os alimentados com $6 \%$ da biomassa ao dia. Os efeitos da temperatura têm sido extensivamente estudados em peixes cultivados, sendo espécie-específicos e dependentes da faixa onde os peixes vivem, como observado por Bermudes et al. (2010), que, ao testarem o efeito de amplo espectro de temperatura no crescimento de Barramundi (Lates calcarifer), também constataram diminuição do crescimento em menores temperaturas. Streit et al. (2010) obtiveram maior crescimento do peixe-rei marinho (Odontesthes argentinensis) - espécie que vive em latitudes maiores do que o robalo-peva - a $23^{\circ} \mathrm{C}$ do que a 26 e $29^{\circ} \mathrm{C}$.

Apesar de ser uma espécie que vive em um amplo espectro de temperatura, o robalo-peva mostrou características de peixe tropical em relação à melhor temperatura para o crescimento, o que indica que a escolha de locais com temperatura média em torno de $28^{\circ} \mathrm{C}$ e a capacidade de manter a temperatura elevada são fatores preponderantes para obtenção de maior crescimento durante a recria.

O menor desempenho produtivo foi obtido nos peixes mantidos a $25^{\circ} \mathrm{C}$, independentemente da taxa de alimentação. Quando observada esta variável, o tratamento mantido nesta temperatura e com $6 \%$ da biomassa ao dia apresentou os menores índices para todos os parâmetros, quando comparado aos demais tratamentos (Tabela 2).

A temperatura influencia, além da demanda alimentar, a partição de energia consumida entre a acumulação de tecido e os sumidouros de energia, como perda de calor, excreção de nitrogênio e fezes (Glencross, 2008). O efeito diferencial da temperatura

Tabela 2. Parâmetros de crescimento e produção de juvenis (média \pm desvio-padrão) de robalo-peva (Centropomus parallelus) durante a primeira fase de crescimento, em diferentes taxas de alimentação e temperaturas.

\begin{tabular}{llccccc}
\hline Temperatura $\left({ }^{\circ} \mathrm{C}\right)$ & Peso $(\mathrm{g})$ & Comprimento $(\mathrm{cm})$ & Conversão alimentar & Ganho de peso $(\mathrm{g})$ & Biomassa final $(\mathrm{g})$ & $\mathrm{TCE}\left({ }^{(1)}(\%)\right.$ \\
\hline & & \multicolumn{5}{c}{$3 \%$ da biomassa ao dia } \\
25 & $17,2 \pm 1,2^{*},+$ & $12,2 \pm 0,3^{*},+$ & $4,4 \pm 0,3+$ & $5,9 \pm 0,7^{*},+$ & $1.034 \pm 70^{*},+$ & $0,70 \pm 0,1^{*},+$ \\
28 & $20,8 \pm 1,9$ & $12,9 \pm 0,4$ & $3,6 \pm 0,4+$ & $8,4 \pm 1,3$ & $1.245 \pm 55$ & $0,86 \pm 0,1$ \\
\hline & & $6 \%$ da biomassa ao dia & \\
25 & $14,3 \pm 0,9^{*}$ & $11,5 \pm 0,3^{*}$ & $12,9 \pm 0,9^{*}$ & $3,6 \pm 0,4^{*}$ & $855 \pm 116^{*}$ & $0,48 \pm 0,1^{*}$ \\
28 & $18,8 \pm 0,4$ & $12,6 \pm 0,3$ & $7,8 \pm 1,0$ & $7,0 \pm 0,8$ & $1.128 \pm 23$ & $0,78 \pm 0,1$ \\
\hline
\end{tabular}

${ }^{(1)} \mathrm{TCE}$, taxa de crescimento específico. *Diferença entre as diferentes temperaturas em uma mesma taxa de alimentação, a $5 \%$ de probabilidade. ${ }^{+}$Diferença entre as diferentes taxas de alimentação em uma mesma temperatura, a 5\% de probabilidade. 
em cada processo fisiológico desempenha papel dominante na determinação da eficiência de utilização dos alimentos. Por exemplo, uma maior eficiência na absorção de ração geralmente é associada a temperaturas mais elevadas. Este resultado foi observado no presente trabalho, uma vez que os peixes criados a $28^{\circ} \mathrm{C}$ apresentaram melhor taxa de conversão alimentar do que os criados a $25^{\circ} \mathrm{C}$. A conversão alimentar aparente variou entre $3,6 \pm 0,4$ e $12,9 \pm 0,9$ e foi significativamente afetada pela taxa de alimentação, pela temperatura e pela interação entre elas. O melhor resultado foi obtido nos peixes alimentados a $3 \%$ da biomassa ao dia a $28^{\circ} \mathrm{C}$, e o pior, nos peixes alimentados a $6 \%$ da biomassa ao dia a $25^{\circ} \mathrm{C}$, quando comparados aos demais tratamentos (Tabela 2). A diminuição da conversão alimentar aparente com o aumento da taxa de alimentação está de acordo com os resultados obtidos por Barbosa et al. (2011b) ao estudar larvas de robalo-peva.

Uma das razões para a baixa conversão alimentar dos peixes no tratamento com $6 \%$ da biomassa ao dia foi a sobra de alimento, provavelmente acima da capacidade de ingestão e saciedade de juvenis desta espécie, já que não foram observadas sobras de alimento nos tanques que receberam $3 \%$ da biomassa ao dia. A sobra de alimento pode ter causado os maiores valores de amônia e, consequentemente, o menor crescimento nos peixes alimentados com a maior quantidade de ração. Isso resulta em aumento nos custos de produção, em razão do desperdício da ração, e na redução da produtividade. Os valores de conversão alimentar obtidos no presente trabalho foram piores que os encontrados por Barbosa et al. (2011a) e por Souza et al. (2011) com juvenis de robalo-peva. Esse resultado provavelmente está relacionado à qualidade da ração oferecida (Souza et al., 2011), formulada para atender às exigências da espécie, enquanto a utilizada no presente trabalho é uma ração comercial, generalista, para espécies carnívoras. Além do tipo de ração, os parâmetros de qualidade de água, especialmente a amônia, e o tamanho inicial dos peixes (Kubitza \& Lovshin, 1999) podem ter influenciado a conversão alimentar.

Os demais parâmetros de produtividade analisados (ganho de peso, biomassa final e taxa de crescimento específico) também foram influenciados pela taxa de alimentação e pela temperatura, e foram significativamente maiores nos peixes alimentados com $3 \%$ da biomassa ao dia a $28^{\circ} \mathrm{C}$. Segundo
Brett \& Groves (1979), o crescimento em peixes é dependente de fatores bióticos, como espécie e estágio de desenvolvimento, e abióticos, como parâmetros de qualidade de água, especialmente a temperatura que aumenta o metabolismo. Esse fato foi observado por Rónyai \& Csengeri (2008) em lúcio-perca (Sander lucioperca), em que os animais criados na temperatura mais alta também apresentaram maior taxa de crescimento específico, mas na maior taxa de alimentação (1,5\%). Saoud et al. (2008) também relataram melhor taxa de crescimento específico a $27^{\circ} \mathrm{C}$ com alimentação à saciedade aparente em "spinefoot rabbitfish" (Siganus rivulatus). A taxa de crescimento específico $(0,86 \%)$ e o ganho de peso $(8,4 \mathrm{~g})$ observados para os peixes alimentados com 3\% da biomassa ao dia a $28^{\circ} \mathrm{C}$ foram ligeiramente superiores aos obtidos por Souza et al. (2011) $(0,77 \%$ e 4,7 g, respectivamente) com juvenis de robalo-peva, na mesma fase de criação, a $25^{\circ} \mathrm{C}$. No entanto, ao se compararem os peixes criados a $25^{\circ} \mathrm{C}$ e $3 \%$ da biomassa ao dia, os resultados de taxa de crescimento específico $(0,70 \%)$ e ganho de peso $(5,9 \mathrm{~g})$ foram mais similares aos encontrados por Souza et al. (2011). A taxa de crescimento específico registrada, nos estudos de recria com robalo-peva, variou de 0,33 a 0,86\% (Ostini et al., 2007; Barbosa et al., 2011a; Souza et al., 2011) e foi inferior à obtida para outras espécies utilizadas ou com potencial para maricultura no Brasil, nesta mesma fase de criação, como o bijupirá (Rachycentrum canadum), com 2,045,4\% ao dia (Resley et al., 2006; Benetti et al., 2010), e a garoupa-verdadeira (Epinephelus marginatus), com aproximadamente $1,7 \%$ ao dia (Ramos et al., 2012). Isso confirma que o robalo-peva é um peixe de crescimento lento, o que exigirá uma série de estudos que visem estabelecer estratégias e produtos que favoreçam o crescimento da espécie. A baixa taxa de crescimento específico é, provavelmente, o principal limitante para o desenvolvimento de um sistema economicamente viável para o robalo-peva.

\section{Conclusões}

1. A produtividade na recria de juvenis de robalo-peva é maximizada a $28^{\circ} \mathrm{C}$, em comparação a $25^{\circ} \mathrm{C}$.

2. A taxa de alimentação a $3 \%$ da biomassa ao dia é mais adequada na recria de juvenis de robalo-peva e 
garante maior produtividade que a alimentação a $6 \%$ da biomassa ao dia.

\section{Agradecimentos}

Ao Ranin Thomé e ao Kaio Lacerda, pelo auxílio na execução do experimento; ao Conselho Nacional de Desenvolvimento Científico e Tecnológico (CNPq) e à Fundação de Amparo à Pesquisa do Espírito Santo (Fapes), pelo apoio financeiro e pela concessão de bolsas.

\section{Referências}

ALVAREZ-LAJONCHÈRE, L.; CERQUEIRA, V.R.; SILVA, I.D.; ARAUJO, J.M.R. Mass production of juveniles of the fat snook Centropomus parallelus in Brazil. Journal of the World Aquaculture Society, v.33, p.506-516, 2002. DOI: 10.1111/ j.1749-7345.2002.tb00031.x.

ALVAREZ-LAJONCHÈRE, L.; TSUZUKI, M.Y. A review of methods for Centropomus spp. (snooks) aquaculture and recommendations for the establishment of their culture in Latin America. Aquaculture Research, v.39, p.684-700, 2008. DOI: 10.1111/j.1365-2109.2008.01921.x.

AMERICAN PUBLIC HEALTH ASSOCIATION. Standard methods for the examination of water and wastewater. $18^{\text {th }}$ ed. Washington: New York, 1992. 1220p.

ANDERSON, M.J.; FAST, A.W. Temperature and feed rate effects on Chinese catfish, Clarias fuscus (Lacepède), growth. Aquaculture Research, v.22, p.435-442, 1991. DOI: 10.1111/ j.1365-2109.1991.tb00756.x.

BALDISSEROTTO, B. Fisiologia de peixes aplicada à piscicultura. 2.ed. Santa Maria: UFSM, 2009. 352p.

BARBOSA, M.C.; JATOBÁ, A.; VIEIRA, F. do N.; SILVA, B.C.; MOURINO, J.L.P.; ANDREATTA, E.R.; SEIFFERT, W.Q.; CERQUEIRA, V.R. Cultivation of juvenile fat snook (Centropomus parallelus Poey, 1860) fed probiotic in laboratory conditions. Brazilian Archives of Biology and Technology, v.54, p.795-801, 2011a. DOI: 10.1590/S1516-89132011000400020.

BARBOSA, M.C.; NEVES, F. de F.; CERQUEIRA, V.R. Taxa alimentar no desempenho de juvenis de robalo-peva em tanquerede. Acta Scientiarum. Animal Sciences, v.33, p.369-372, 2011b. DOI: 10.4025/actascianimsci.v33i4.1141.

BENETTI, D.; SARDENBERG, B.; HOENIG, R.; WELCH, A.; STIEGLITZ, J.; MIRALAO, S.; FARKAS, D.; BROWN, P.; JORY, D. Cobia (Rachycentron canadum) hatchery-to-market aquaculture technology: recent advances at the University of Miami Experimental Hatchery (UMEH). Revista Brasileira de Zootecnia, v.39, p.60-67, 2010. DOI: 10.1590/S1516-35982010001300008.

BERMUDES, M.; GLENCROSS, B.; AUSTEN, K.; HAWKINS, $\mathrm{W}$. The effects of temperature and size on the growth, energy budget and waste outputs of barramundi (Lates calcarifer). Aquaculture, v.306, p.160-166, 2010. DOI: 10.1016/j.aquaculture.2010.05.031.
BRETT, J.R.; GROVES, T.D.D. Physiological energetics. Fish physiology: Bioenergetics and Growth, v.8, p.279-352, 1979. DOI: 10.1016/S1546-5098(08)60029-1.

CERQUEIRA, V.R. Cultivo de robalo-peva (Centropomus parallelus). In: BALDISSEROTTO, B.; GOMES, L. de C. (Ed.). Espécies nativas para piscicultura no Brasil. 2.ed. Santa Maria: UFSM, 2010. p.489-520.

CERQUEIRA, V.R.; TSUZUKI, M.Y. A review of spawning induction, larviculture, and juvenile rearing of the fat snook, Centropomus parallelus. Fish Physiology Biochemistry, v.35, p.17-28, 2009. DOI: 10.1007/s10695-008-9245-y.

FERRAZ, E. de M.; CARVALHO, C.G.S.; SCHAEFER A.L.C.; NARAHARA, M.Y.; CERQUEIRA, V.R. Influência da temperatura de cultivo sobre crescimento e diferenciação sexual de robalo-peva, Centropomus parallelus Poey, 1860. Revista Brasileira de Engenharia de Pesca, v.6, p.1-16, 2011.

GLENCROSS, B.D. A factorial growth and feed utilization model for barramundi, Lates calcarifer based on Australian production conditions. Aquaculture Nutrition, v.14, p.360-373, 2008. DOI: 10.1111/j.1365-2095.2007.00543.x.

KUBITZA, F.; LOVSHIN, L.L. Formulated diets, feeding strategies and cannibalism control during intensive culture of juvenile carnivorous fishes. Reviews in Fisheries Science, v.7, p.1-22, 1999. DOI: 10.1080/10641269991319171.

LEMARIÉ, G.; DOSDAT, A.; COVÈS, D.; DUTTO, G.; GASSET, E.; PERSON-LE RUYET, J. Effect of chronic ammonia exposure on growth of European seabass (Dicentrarchus labrax) juveniles. Aquaculture, v.229, p.479-491, 2004. DOI: 10.1016/ S0044-8486(03)00392-2.

OSTINI, S.; OLIVEIRA, I. da R.; SERRALHEIRO, P.C. da S.; SANCHES, E.G. Criação do robalo-peva ("Centropomus parallelus") submetido a diferentes densidades de estocagem. Revista Brasileira de Saúde e Produção Animal, v.8, p.250-257, 2007.

RAMOS, F.M.; SANCHES, E.G.; FUJIMOTO, R.Y.; COTTENS, K.F.; CERQUEIRA, V.R. Crescimento de juvenis de garoupa-verdadeira Epinephelus marginatus submetidos a diferentes dietas. Boletim do Instituto de Pesca, v.38, p.81-88, 2012.

RANDALL, D.J.; TSUI, T.K.N. Ammonia toxicity in fish. Marine Pollution Bulletin, v.45, p.17-23, 2002. DOI: 10.1016/ S0025-326X(02)00227-8.

RESLEY, M.J.; WEBB JUNIOR, K.A.; HOLT, G.J. Growth and survival of juvenile cobia, Rachycentron canadum, at different salinities in a recirculating aquaculture system. Aquaculture, v.253, p.398-407, 2006. DOI: 10.1016/j.aquaculture.2005.08.023.

RIBEIRO, F.F.; TSUZUKI, M.Y. Compensatory growth responses in juvenile fat snook, Centropomus parallelus Poey, following food deprivation. Aquaculture Research, v.41, p.226-233, 2010. DOI: 10.1111/j.1365-2109.2010.02507.x.

RIVAS, L.R. Systematic review of the perciform fishes of the genus Centropomus. Copeia, n.3, p.579-611, 1986. DOI: $10.2307 / 1444940$. 
ROCHA, A.J. da S.; GOMES, V.; VAN NGAN, P.; PASSOS, M.J. de A.C.R.; FURIA, R.R. Metabolic demand and growth of juveniles of Centropomus parallelus as function of salinity. Journal of Experimental Marine Biology and Ecology, v.316, p.157-165, 2005. DOI: 10.1016/j.jembe.2004.11.006.

RÓNYAI, A.; CSENGERI, I. Effect of feeding regime and temperature on ongrowing results of pikeperch (Sander lucioperca L.). Aquaculture Research, v.39, p.820-827, 2008. DOI: 10.1111/j.1365-2109.2008.01935.x.

SAOUD, I.P.; MOHANNA, C.; GHANAWI, J. Effects of temperature on survival and growth of juvenile spinefoot rabbitfish (Siganus rivulatus). Aquaculture Research, v.39, p.491-497, 2008. DOI: 10.1111/j.1365-2109.2007.01903.x.

SILVA, C.R.; GOMES, L.C.; BRANDÃO, F.R. Effect of feeding rate and frequency on tambaqui (Colossoma macropomum) growth, production and feeding costs during the first growth phase in cages. Aquaculture, v.264, p.135-139, 2007. DOI: 10.1016/j. aquaculture.2006.12.007.

SOUZA, J.H. de; FRACALOSSI, D.M.; GARCIA, A.S.; RIBEIRO, F.F.; TSUZUKI, M.Y. Desempenho zootécnico e econômico de juvenis de robalo-peva alimentados com dietas contendo diferentes concentrações proteicas. Pesquisa Agropecuária Brasileira, v.46, p.190-195, 2011. DOI: 10.1590/S0100-204X2011000200011.

STREIT, D.P.; TESSER, M.B.; BURKERT, D.; CASTAÑO SANCHEZ, C.; SAMPAIO, L.A. Survival and growth of juvenile marine pejerrey, Odontesthes argentinensis, reared at different temperatures. Journal of the World Aquaculture Society, v.41, p.931-935, 2010. DOI: 10.1111/j.1749-7345.2010.00436.x.

TSUZUKI, M.Y.; SUGAI, J.K.; MACIEL, J.C.; FRANCISCO, C.J.; CERQUEIRA, V.R. Survival, growth and digestive enzyme activity of juveniles of the fat snook (Centropomus parallelus) reared at different salinities. Aquaculture, v.271, p.319-325, 2007. DOI: 10.1016/j.aquaculture.2007.05.002.

XIE, S.; ZHENG, K.; CHEN, J.; ZHANG, Z.; ZHU, X.; YANG, Y. Effect of water temperature on energy budget of Nile tilapia, Oreochromis niloticus. Aquaculture Nutrition, v.17, p.683-690, 2011. DOI: 10.1111/j.1365-2095.2010.00827.x.

ZAR, J.H. (Ed.). Biostatistical analysis. $4^{\text {th }}$ ed. Prentice Hall: Upper Saddle River, 1999. 663p.

$\overline{\text { Recebido em } 15 \text { de julho de } 2011 \text { e aprovado em } 14 \text { de maio de } 2012}$ 